\title{
Energy processes with natural quantization
}

\author{
E. A. Shakhova ${ }^{1}$, P. P. Rymkevich ${ }^{1}, A$. S. Gorshkov ${ }^{2}, M . Y$. Egorov ${ }^{3, *}$, and A. S. Stepashkina ${ }^{4}$ \\ ${ }^{1}$ A.F. Mozhaysky Military Space Academy, St. Petersburg, Russia \\ ${ }^{2}$ PAO Gazprom, St. Petersburg, Russia \\ ${ }^{3}$ Peter the Great St. Petersburg Polytechnic University, St. Petersburg, Russia \\ ${ }^{4}$ Saint Petersburg State University of Aerospace Instrumentation, St. Petersburg, Russia
}

\begin{abstract}
The paper shows that the quantum-mechanical approach is applicable to most macro processes occurring in nature include the power industry. The mathematical apparatus of the isomorphic Heisenberg algebra is proposed. A non-commutative ring is constructed within which the commutation relations are given. The transition from quantum to classical theory is shown.
\end{abstract}

\section{Introduction}

Most of the processes in nature including energy processes are random. Random processes are usually described by quantum theory at the micro level. Modern methods of the processes describing associated with the exchange and energy transfer require new mathematical approaches. We offer a new mathematical approach based on isomorphic Heisenberg algebra. A noncommutative ring is constructed within which the commutation relations are given.

\section{Mathematical apparatus: non- commutative ring}

We understand 'the process' as an ordered sequence of events characterized by a set of specific numerical values. Let us consider the set of functions $C_{t, \tau}$ of two real variables $t$ and $\tau$. Functions are defined and piecewise continuous for $\tau \geq 0$ and analytic in a variable $t \in(-\infty ;+\infty)$. Let us assume that $a(t, \tau), b(t, \tau), c(t, \tau) \in C_{t, \tau}$. We define the multiplication of functions as follows:

$$
\begin{aligned}
& c(t, \tau)=a(t, \tau) \bar{\otimes} b(t, \tau) \stackrel{\text { Def }}{=} \\
& =\int_{0}^{\tau} a\left(t, \tau_{1}\right) b\left(t+\tau_{1}, \tau-\tau_{1}\right) d \tau_{1}
\end{aligned}
$$

Have $R_{\bar{\otimes}}$ as the ring of functions $C_{t, \tau}$ with a natural definite addition and multiplication in the sense of (1).

We consider an ordered sequence of functions $a, b, c \ldots \in R_{\bar{\otimes}}$. In some tasks $a(t, \tau)$ can be interpreted as a distribution function of some event $\mathrm{A}$ which began at the time $t$ and is distributed over the event duration $\tau \geq 0$. In this case the above sequence of functions is called a process which consist of the sequential execution of events $\mathrm{A}, \mathrm{B}, \mathrm{C} \ldots$. . Hereinafter the elements of the ring $R_{\bar{\otimes}}$ are functions of the corresponding events. The need to use the ring $R_{\bar{\otimes}}$ means that most of the nature phenomena around us are some ordered chains of certain events.

Here is an example. Let the process $\mathrm{E}$ is described by the function $e(t, \tau)$. It consists in the implementation of two ordered events $\mathrm{A}$ and $\mathrm{B}$ or $\mathrm{C}$ and $\mathrm{D}$ which described by the functions a, b, c, d respectively. Then we have

$$
e(t, \tau)=a(t, \tau) \bar{\otimes} b(t, \tau)+c(t, \tau) \bar{\otimes} d(t, \tau)
$$

Note that the probability amplitudes in a quantum mechanics are summarized according to the same principle.

In the simplest case, if $a, b, c \ldots$ don't depend on the variable $t$ explicitly (for example, the start time of an event) then the integral (1) represents the usual convolution of functions (multiplication in the sense of Mikusinski [1]), i.e. $c(\tau)=a(\tau) * b(\tau)$. In this way $R_{*} \subset R_{\bar{\otimes}}$. Note that the ring has zero divisors unlike the Mikusinsky ring, i.e. it can't be extended in an elementary way to the relationship field. A ring $R_{\bar{\otimes}}$ as like a ring, $R_{*}$ is a ring with a unit moreover the zero of a single element is the Dirac delta function $\delta(\tau)$. As it's customary in operational calculus [2, 3], elements of the ring $R_{\bar{\otimes}}$ are called operators (distributions). And generalized functions are also included in the class of functions $C_{t, \tau}$. In the future it is more convenient to use not the ring $R_{\bar{\otimes}}$ itself but the ring $R_{\otimes}$. Isomorphism

\footnotetext{
*Corresponding author: mikhail.yu.egorov@gmail.com
} 
between rings $R_{\bar{\otimes}}$ and $R_{\otimes}$ is established using the Laplace transform

$$
A(t, s)=\int_{0}^{\infty} a(t, \tau) e^{-\tau s} d \tau
$$

where $A(t, s)$ is the analytic function on both variable (real $t$ and imaginary $s$ ).

We define the multiplication of functions as $A(t, s) \otimes B(t, s)=C(t, s) ; A, B, C \in R_{\otimes}$, it describe a multiplicative semigroup of a ring $R_{\otimes}$ like this

$$
\begin{aligned}
& C(t, s) \stackrel{\text { Def }}{=} \sum_{n=0}^{\infty} \frac{(-1)^{n}}{n !} \cdot \frac{\partial^{n} A(t, s)}{\partial s^{n}} \cdot \frac{\partial^{n} B(t, s)}{\partial t^{n}}= \\
& =A(t, s) \otimes B(t, s)
\end{aligned}
$$

Indeed, let $\mathrm{A}(\mathrm{t}, \mathrm{s})$ and $\mathrm{B}(\mathrm{t}, \mathrm{s})$ are the Laplace images of the distributions $a(t, \tau)$ and $b(t, \tau), C(t, s)$ is the Laplace image $c(t, \tau)=a(t, \tau) \bar{\otimes} b(t, \tau)$, then

$$
\begin{aligned}
& C(t, s)=\hat{L}\left\{\int_{0}^{\tau} a\left(t, \tau_{1}\right) b\left(t+\tau_{1}, \tau-\tau_{1}\right) d \tau_{1}\right\}= \\
& =\hat{L}\left\{\int_{0}^{\tau} a\left(t, \tau_{1}\left[\sum_{n=0}^{\infty} \frac{1}{n !} b_{t}^{(n)}\left(t, \tau-\tau_{1}\right) \tau_{1}^{n}\right] d \tau_{1}^{n}\right\}=\right. \\
& =\hat{L}\left\{\sum_{n=0}^{\infty} \int_{0}^{\tau}\left[a\left(t, \tau_{1}\right) \tau_{1}^{n}\right] \cdot b_{t}^{(n)}\left(t, \tau-\tau_{1}\right) d \tau_{1}\right\}= \\
& =\sum_{n=0}^{\infty} \frac{1}{n !}\left\{\hat{L}\left[a(t, \tau) \tau^{n}\right]\right\} \cdot\left\{\hat{L} b_{t}^{(n)}(t, \tau)\right\}= \\
& =\sum_{n=0}^{\infty} \frac{(-1)^{n}}{n !} A_{s}^{(n)}(t, s) \cdot B_{t}^{(n)}(t, s)
\end{aligned}
$$

The associativity of the ring $R_{\otimes}$ follows from the associativity of the ring $R_{\bar{\otimes}}$. Multiplication in the sense of (2) is connected with the addition of the distributive laws. $R_{\otimes}$ is a ring with a unit, moreover the unit of the $\operatorname{ring} R_{\otimes}$ is a function $E(t, s) \equiv 1$.

\section{Ring properties}

Let's consider some of the simplest properties of the ring $R_{\otimes}$.

$$
\begin{aligned}
& \text { 1. }[t, s]=t \otimes s-s \otimes t=1 \\
& \text { 2. } \begin{aligned}
{[s, A(t, s)] } & =-A_{t}^{\prime}(t, s) \\
{[t, A(t, s)] } & =A_{s}^{\prime}(t, s)
\end{aligned} \text {. }
\end{aligned}
$$

Thus the differentiation operation of the analytic function $A(t, s)$ in one of the variables is reduced to taking the corresponding switch. It should be noted that, in contrast to classical quantum mechanics, $t$ and $s$ are not operators but numerical variables. $A(t, s)$ is an analytic function of numerical variables.

The rules for differentiating multiplication in the sense of (2) are the same as for ordinary multiplication, i.e.

$$
\begin{aligned}
& \text { 3. } \frac{\partial}{\partial s}[A(t, s) \otimes B(t, s]= \\
& =A_{s}^{\odot}(t, s) \otimes B(t, s)+A(t, s) \otimes B_{s}^{\odot}(t, s) \\
& \frac{\partial}{\partial t}[A(t, s) \otimes B(t, s]= \\
& =A_{t}^{\oplus}(t, s) \otimes B(t, s)+A(t, s) \otimes B_{t}^{\odot}(t, s)
\end{aligned}
$$

Multiplication in the sense of (2) is closely related to the differentiation operator

$$
\begin{gathered}
\text { 4. } \begin{array}{c}
A(t, s) \otimes B(t, s)=A\left(t, S-D_{t}\right) \cdot B(t, s)= \\
=B\left(t-D_{S}, S\right) \cdot A(t, s)
\end{array} \\
\text { where } D_{t} \equiv \frac{\partial}{\partial t} \quad D_{s} \equiv \frac{\partial}{\partial s} .
\end{gathered}
$$

The numbers above the non-commuting elements indicate the order in which they follow from right to left. This designation was suggested by V.P. Maslov in [4]. For the first time, apparently, Feyman [5] began to systematically use functions of ordered operators [6-9] as well as calculus of Weyl functions $[10,11]$. Multiplication in the sense of (2) is closely related to the Heisenberg algebra $[12,13]$. The main difference of the considered device is that all elements of the rings $R_{\bar{\otimes}}$ and $R_{\otimes}$ are the usual functions of numerical variables and, consequently, gives them a different physical meaning. A detailed presentation of this method is considered in the work of the authors [14].

\section{Application of mathematical approach}

There is a significant number of works devoted to obtaining various commutation relations and action rules from non-commuting operators [4, 7-10]. A summary of the non-commutative analysis formulas is given in [6].

A ring $R_{\otimes}$ using the Laplace transform is convenient for a number of practical problems with the given initial conditions. However, to solve common physical problems it is preferable to use the exponential Fourier transform instead of the Laplace. In this case if we put $s=i \omega$ then all the above relations will remain in force and the expression (2) will be rewritten as 


$$
\begin{gathered}
C(t, \omega)=A(t, \omega) \otimes B(t, \omega)= \\
=\sum_{n=0}^{\infty} \frac{(i)^{n}}{n !} A_{\omega}^{(n)}(t, \omega) B_{t}^{(n)}(t, \omega), \\
c(t, \tau)=a(t, \tau) \bar{\otimes} b(t, \tau)= \\
=\int_{-\infty}^{+\infty} a\left(t, \tau_{1}\right) b\left(t+\tau_{1}, \tau-\tau_{1}\right) d \tau_{1} .
\end{gathered}
$$

Here $A, B, C$ are Fourier images of distributions $a, b$, $c$ on the variable $\tau$.

Usually in physical processes we understand the variable $t$ as time and the variable $\omega$ as the event frequency.

We use $\varepsilon=h \omega$ instead of $\omega$ which is formally called quasi-energy. /it is known from quantum mechanics that $\hbar=1,05 \cdot 10^{-34} \mathrm{~J} / \mathrm{s}$. In variables $t, \varepsilon$ expression (3) takes the form

$$
\begin{aligned}
& c(t, \varepsilon)=A(t, \varepsilon) \otimes B(t, \varepsilon)= \\
& =\sum_{n=0}^{\infty} \frac{(i \hbar)^{n}}{n !} A_{\varepsilon}^{(n)}(t, \varepsilon) B_{t}^{(n)}(t, \varepsilon)
\end{aligned}
$$

Wherein

$$
[\varepsilon, t]=i \hbar
$$

Make a formal transition in relation (5) then multiplication in the sense of (5) turns into ordinary commutative multiplication, i.e.

$$
c(\underset{\hbar \rightarrow 0}{c})=A(t, \varepsilon) \cdot B(t, \varepsilon)
$$

In the space of the originals this limiting passage means that

$$
\begin{aligned}
& c(t, \varepsilon)=\int_{0}^{\tau} a\left(t, \tau_{1}\right) \cdot b\left(t, \tau-\tau_{1}\right) d \tau_{1}= \\
& =a(t, \tau) * b(t, \tau) \text {. }
\end{aligned}
$$

The latter means that it neglects the change of properties during a long event. For a quantum mechanical description, this transition means a transition to a classical description.

All of the above indicates that most of the phenomena occurring in nature are naturally quantized since for its description it is necessary to use a noncommutative ring $R_{\otimes}$. Philosophically, this statement means that a part of quantum-mechanical statements is valid not only for micro-objects (quantummechanical objects) but also for the macro-objects.

A ring similar to $R_{\otimes}$ can be constructed for any pair of real variables defined on the whole numerical axis. Let be $a\left(x, r_{x}\right), b\left(x, r_{x}\right), c\left(x, r_{x}\right) \in R$ the set of functions which are analytic in the variable $\mathrm{x}$ and piecewise continuous in the variable $r_{x}$ with natural addition and multiplication in the sense of (7).

$$
\begin{aligned}
& c\left(x, r_{x}\right)=a\left(x, r_{x}\right) \bar{\Delta} b\left(x, r_{x}\right)= \\
& =\int_{-\infty}^{+\infty} a(x, \theta) b\left(x+\theta, r_{x}-\theta\right) d \theta .
\end{aligned}
$$

In some cases the expression (7) can be interpreted as two consecutive events combined into one and consisting in the transition of "something" from a point with $\mathrm{x}$-coordinate to a point with a coordinate $x+r_{x}$ (i.e. $r_{x}$ plays the role of a variable). In this case the first event is the movement from point $\mathrm{x}$ to $\theta$ and the second event is the movement from point with coordinate $x+\theta$ to $r_{x}-\theta$. Under probabilistic interpretation the expression (7) is the well-known SmoluchowskiKolmogorov-Chapman equation. We use the Fourier transform, i.e.

$$
A\left(x, k_{x}\right)=\int_{-\infty}^{+\infty} a\left(x, r_{r}\right) e^{i k_{x} r_{x}} d r_{x}
$$

Define the multiplication $c\left(x, k_{x}\right)=A\left(x, k_{x}\right) \Delta B\left(x, k_{x}\right), A, B, C \in R_{\Delta}$, than we have

$$
\begin{aligned}
& c\left(x, k_{x}\right)=\hat{F}\left[\int_{-\infty}^{+\infty} a(x, \theta) b\left(x+\theta, r_{x}-\theta\right) d \theta\right]= \\
& =\hat{F}\left[\int_{-\infty}^{+\infty} a(x, \theta) \sum_{n=0}^{\infty} \frac{\theta^{n}}{n !} b_{x}^{(n)}\left(x, r_{x}-\theta\right) d \theta\right]= \\
& =\sum_{n=0}^{\infty} \frac{1}{n !} \hat{F}\left\{\int_{-\infty}^{+\infty}\left[a(x, \theta) \theta^{n}\right] b_{x}^{(n)}\left(x, r_{x}-\theta\right) d \theta\right\}= \\
& =\sum_{n=0}^{\infty} \frac{(-i)^{n}}{n !} A_{k_{x}}^{(n)}\left(x, k_{x}\right) B_{x}^{(n)}\left(x, k_{x}\right) .
\end{aligned}
$$

The properties of the ring $R_{\Delta}$ are completely equivalent to the properties of the ring $R_{\otimes}$. It's important to mention that

$$
\left[k_{x}, x\right]=k_{x} \Delta x-x \Delta k_{x}=-i .
$$

Multiplication in the sense of (8) is easily generalized to the case of discrete variables' functions. Note that all of the above can be generalized to any finite number of real variables. In particular for the case of three independent variables in the ring $R_{\Delta}$ we define the multiplication in a similar way: 


$$
\begin{aligned}
& c(\vec{R}, \vec{r})=a(\vec{R}, \vec{r}) \bar{\Delta} b(\vec{R}, \vec{r}) \stackrel{\text { Def }}{=} \\
& =\iint_{-\infty}^{+\infty} \int a\left(x, y, z \mid \theta_{x}, \theta_{y}, \theta_{z}\right) \cdot b\left(x+\theta_{x}, y+\right. \\
& \left.+\theta_{y}, z+\theta_{z} \mid r_{x}-\theta_{x}, r_{y}-\theta_{y}, r_{z}-\theta_{z}\right) \cdot d \theta_{x} d \theta_{y} d \theta_{z}
\end{aligned}
$$
have:

Then for the corresponding Fourier transforms we

$$
\begin{aligned}
& -(\vec{R}, \vec{k})=A(\bar{R}, \bar{k}) \Delta B(\vec{R}, \vec{k})= \\
& \sum_{\substack{n_{x}=0 \\
n_{y}=0 \\
n_{z}=0}}^{\infty} \frac{(-i)^{n_{x}+n_{y}+n_{z}}}{n_{x} ! n_{y} ! n_{z} !} \cdot \frac{\partial^{n_{x}+n_{y}+n_{z}} A(\bar{R}, \bar{k})}{\partial k_{x}^{n_{x}} \partial k_{y}^{n_{y}} \partial k_{z}^{n_{z}}} \cdot \frac{\partial B^{n_{x}+n_{y}+n_{z}}(\bar{R}, \bar{k})}{\partial x_{x}^{n_{x}} \partial y_{y}^{n_{y}} \partial z_{z}^{n_{z}}},
\end{aligned}
$$

where $\vec{R}\{x, y, z\}, \vec{k}\left\{k_{x}, k_{y}, k_{z}\right\}$ - vectors of a linear vector space (over a field c - complex numbers).

We introduce a quantity $\vec{P}=h \vec{k}$ called further a quasi-momentum. It shouldn't be confused with the concept of "quasi-momentum" in solid state physics. In variables $\vec{R}$ and $\vec{P}$ the expression (12) takes the form:

$$
\begin{aligned}
& C(\vec{R}, \vec{p})=A(\vec{R}, \vec{p}) \Delta B(\vec{R}, \vec{p})= \\
& \sum_{\substack{n_{x}=0 \\
n_{y}=0 \\
n_{z}=0}}^{\infty} \frac{(-i \hbar)^{n_{x}+n_{y}+n_{z}}}{n_{x} ! n_{y} ! n_{z} !} \cdot \frac{\partial^{n_{x}+n_{y}+n_{z}} A(\vec{R}, \vec{p})}{\partial p_{x}^{n_{x}} \partial p_{y}^{n_{y}} \partial p_{z}^{n_{z}}} \cdot \frac{\partial^{n_{x}+n_{y}+n_{z}} B(\vec{R}, \vec{k})}{\partial x_{x}^{n_{x}} \partial y_{y}^{n_{y}} \partial z_{z}^{n_{z}}} .
\end{aligned}
$$

Note that for the quasi-momentum as for the quantum mechanical momentum the Heisenberg commutation relations is held and can be written as:

$$
\begin{aligned}
& {\left[p_{x}, x\right]=-i \hbar} \\
& {\left[p_{y}, y\right]=-i \hbar} \\
& {\left[p_{z}, z\right]=-i \hbar}
\end{aligned}
$$

It is especially important to note that the mentioned commutation relations don't connect with the "physics" of any processes; it is only a description method.

In the physics of processes it is convenient to use the ring Ro where the multiplication is defined as $(\circ=\Delta \otimes)$ :

$$
\begin{aligned}
& c(t, \vec{R} \mid \vec{p}, \varepsilon)=A(t, \vec{R} \mid \vec{P}, \varepsilon) \bar{\circ} B(t, \vec{R} \mid \vec{P}, \varepsilon)= \\
& =\sum_{\substack{n=0 \\
n_{x}=0 \\
n_{y}=0 \\
n_{z}=0}}^{\infty}\left(\frac{(i \hbar)^{n} \cdot(-i \hbar)^{n_{x}+n_{y}+n_{z}}}{n ! n_{x} ! n_{y} ! n_{z} !} \cdot \frac{\partial^{n+n_{y}+n_{y}+n_{z}} A(t, \vec{R} \mid \vec{P}, \varepsilon)}{\partial \varepsilon^{n} \partial p_{x}^{n_{x}} \partial p_{y}^{n_{y}} \partial p_{z}^{n_{z}}}\right. \\
& \left.\cdot \frac{\partial^{n+n_{x}+n_{y}+n_{z}} B(t, \vec{R} \mid \vec{P}, \varepsilon)}{\partial t^{n} \partial x_{x}^{n_{x}} \partial y_{y}^{n_{y}} \partial z_{z}^{n_{z}}} .\right)
\end{aligned}
$$

In the space of the originals this multiplication has the form

$$
\begin{aligned}
& c(t, \vec{R} \mid \vec{\theta}, \tau)=a(t, \vec{R} \mid \vec{\theta}, \tau) \circ b(t, \vec{R} \mid \vec{\theta}, \tau) \stackrel{\text { Def }}{=} \\
& \iiint \int(a(t, \vec{R} \mid \vec{\eta}, \xi) \cdot \\
& \cdot b(t+\xi, \vec{r}+\vec{\eta} \mid \vec{\theta}-\vec{y}, \tau-\xi)) d \xi d \eta_{x} d \eta_{y} d \eta_{z}
\end{aligned}
$$

which can be interpreted as a transition from one point of four-dimensional space to another.

Usually all elements $A, B, C \ldots \in R$ form an algebra with unity (over the field of complex numbers in general). Most often the elements A, B, C ... are square matrices. In this case the unit element of the algebra $\mathrm{R}$ will be the identity matrix.

\section{Conclusion}

Thus, it was proposed the mathematical approach of isomorphic Heisenberg algebra with a non-commutative ring. Commutation relations were also given. The transition from quantum to classical theory is shown. More than that it can be shown that to describe most of the processes in nature, it is necessary to adopt the quantum approach.

\section{References}

[1] Y. Mikusinski, Operator calculation (Moscow: Il) (1956)

[2] V.A. Ditkin, A.P. Prudnikov, Operator calculation rogress (Moscow: Vishnaya shkola) (1957)

[3] G. Beithman, A. Erdein, Tables of integral transformations (Moscow: Nauka) (1969)

[4] V.P. Maslov, Operator methods (Moscow: Nauka) (1973)

[5] R.P. Feyman, Problems of modern physics 3, 37-79 (1955)

[6] M.V. Karasev, V.P. Maslov, Non-linear Poisson brackets. Geometry quantization (Moscow: Nauka) (1991)

[7] M.V. Karasev, V.P. Maslov, Modern problems of mathematics 13, 145-267 (1979)

[8] V.P. Maslov, TNF 3(2), 185-209 (1977)

[9] V.P. Maslov, V.E. Nezaykinsky, Modern problems of mathematics 13, 144

[10] M.V. Karasev, Mathematical Notes 26(6), 885-907 (1979)

[11] R.F.V. Anderson, I. Funct. Anal. 4(2), 240267 (1969)

[12] F.A. Berezin, Izvestiya Academy of Sciences of the USSR 38(5), 1116-1175 (1974)

[13] F.A. Berezin, Izvestiya Academy of Sciences of the USSR 39(2), 363-402 (1975)

[14] P.P. Rymkevich, A.S. Gorshkov, Transfer theory (SPb: Polytechnic University Press) (2015) 